DOI: http://dx.doi.org/10.22201/iie.18703062e.2000.77.1949

\author{
MICHAEL DREWES
}

\title{
Otra aproximación a Carl Gangolf Kaiser (1837-1895), arquitecto de la corte de emperador Maximiliano ${ }^{\mathrm{I}}$
}

El archiduque Fernando M aximiliano de $\mathrm{H}$ absburgo

Y

A EL JOVEn ARChIDUQue Fernando M aximiliano de H absburgo (I832-I867) poseía un individualismo marcado; a la edad de diecisiete años había construido en $\mathrm{H}$ ietzing, fuera del parque imperial de Schönbrunn, una casa con jardín, de acuerdo con su imaginación y al estilo suizo que llamó M axing. En este caso actuaba, aunque en cierta medida humilde, como mecenas artístico. También emprendió experimentos propios en el modelado y en la pintura, demostrando bastante diligencia en el retrato. Su vivo interés por las artes salió a flote en todas las etapas de su vida. ${ }^{2}$ Aún de niño, el archiduque encontró placer en las artes. Se cuenta cómo había salido con su aya a ver una exposición y quiso adquirir una pintura de D affinger a toda costa, aunque el precio superó sus posibilidades económicas. ${ }^{3}$

I. Ponencia presentada en el seminario internacional La D efinición del Estado $\mathrm{N}$ acional M exicano, I857-1867 el 6 de agosto de 1998 en el Archivo General de la N ación, Palacio de Lecumberri.

2. Elisabeth Springer, "M aximilians Persönlichkeit", en M aximilian von M exiko I832-I867, 1974, p. ז3. También M aus Eggert, "M aximilian und seine Kunstschöpfungen", ibidem, p. 72.

3. W erner K itlitschka, "Erzherzog Ferdinand M aximilian und die bildende K unst", ibidem, 1974 , p. 58. 
DOI: http://dx.doi.org/10.22201/iie.18703062e.2000.77.1949

152

MICHAEL DREWES

Como típico europeo ilustrado del siglo xIX, M aximiliano era un amante de las bellas artes. El antes mencionado proyecto $M$ axing, una casa de madera en un parque de Viena que hoy en día ya no existe, era una muestra de su talento para la arquitectura, que culminó con los diseños del arquitecto $C$ arl Junker para el palacio de M iramar y con los jardines y el palacio de Lacroma en la costa del $\mathrm{M}$ ar Adriático, proyectados por el arquitecto Julius $\mathrm{H}$ ofmann, que no fueron realizados. ${ }^{4}$

Los escasos tres años que duró el Segundo Imperio no le dieron tiempo para desenvolverse en las artes. Hubo proyectos arquitectónicos que, podemos preciarnos, no se realizaron; la mayor parte de ellos se quedó en el boceto. No obstante, podemos cel ebrar como un verdadero descubrimiento el haber hallado planos y bosquejos de proyectos elaborados por un arquitecto austriaco, Carl G angolf Kaiser, y por otros colegas suyos, mexicanos, como el arquitecto Ramón Rodríguez Arrangoiti y el ingeniero Eleuterio M éndez. ${ }^{5}$

\section{Biografía del arquitecto Carl Gangolf Kaiser}

El arquitecto Carl G angolf Kaiser nació el I2 de febrero de ı837 en Viena, estudió arquitectura en su ciudad natal y en Munich bajo la conducción de Ludwig Lange. $\mathrm{H}$ izo varios viajes por Europa, América del N orte y Centroamérica. Fue comisionado en I864 por el emperador M aximiliano como arquitecto de la corte para los trabajos de restauración y remodelación del Palacio Nacional y del castillo de Chapultepec en la ciudad de M éxico, y del Palacio de Cortés en Cuernavaca. D espués de la muerte de M aximiliano regresó a su patria, donde trabajó principalmente para la nobleza. Remodeló el castillo medieval de K reuzenstein a partir de i874; el de $\mathrm{H}$ ardegg, a partir de I878, junto con Humbert Walcher, caballero de Moltheim;' ${ }^{6}$ restauró el de Liechtenstein, cerca de M ödling. M odificó el palacio barroco de Auersberg a partir de 1880 y efectuó obras menores en el de Kinsky en i869 (ambos en la

4. Ferdinand Anders, D ie Gärten M aximilians, Viena, 1987, pp. 22-23.

5. Agradezco al profesor Jorge O Ivera H ernández la información sobre la existencia de planos arquitectónicos en el archivo del Instituto de G eografía y M eteorología de Tacubaya.

6. Franz M üllner, "J ohann Carl Fürst K hevenhüller-M etsch, ein Kampfgefährte Kaiser M a ximilians von M exiko", en M aximilian von M exiko I832-I867, op. cit., p. I55. 


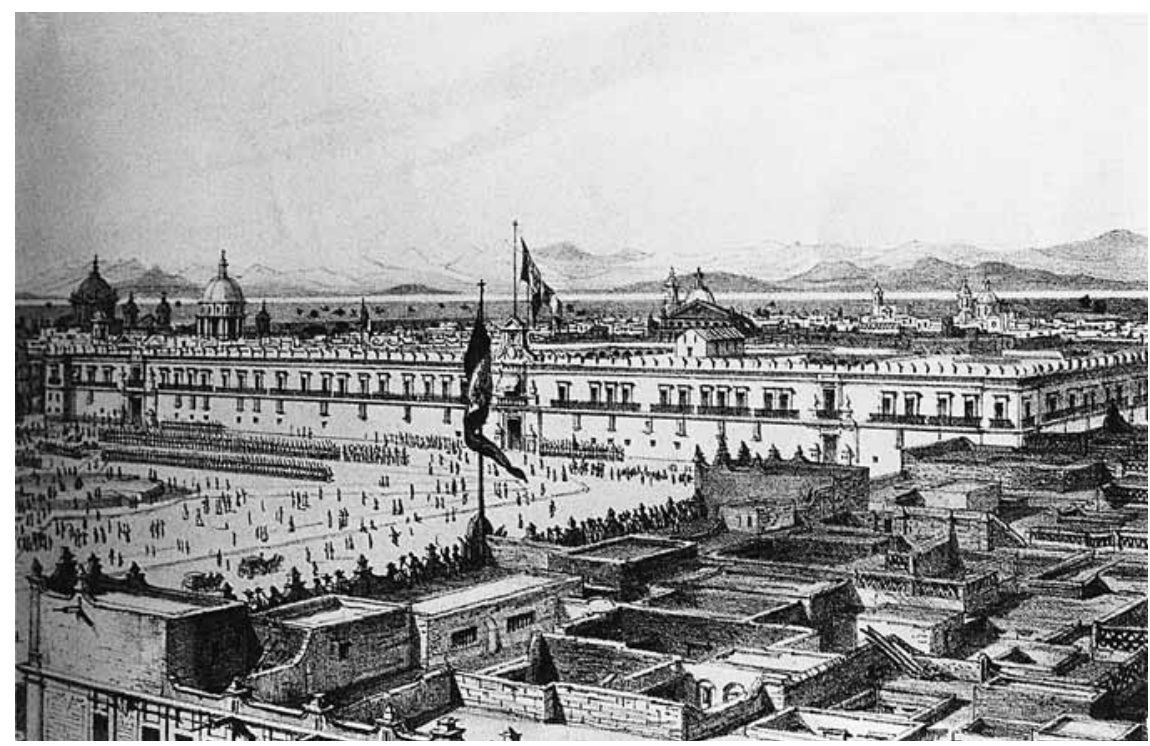

Figura i. El Zócalo de la ciudad de M éxico, litografía de D ecaen, I856.

ciudad de Viena), y en el castillo de Seebarn, a orillas del D anubio. M urió el 2 de septiembre de 1895 en el manicomio de Inzersdorf, cerca de V iena. ${ }^{7}$

Todavía antes de irse a M éxico, el archiduque Fernando M aximiliano pensaba establecer una galería de artes en el palacio de $\mathrm{M}$ iramar a orillas del $M$ ar Adriático, y en un escrito dictado por él en Chapultepec en i866 se designó una comisión compuesta por el prefecto de M iramar, el arquitecto Carl Gangolf Kaiser, los profesores Bilimek y Simón Leo Reinisch, el doctor Ilek y los pintores Fiedler y Josef Selleny (I824-1875). ${ }^{8}$

El doctor Eckart Vancsa del Bundesdenkmalamt u O ficina de Restauración de Viena investigó la biografía de Kaiser, y la corrigió en el sentido de que Kaiser no estudió con el pintor paisajista Julius Lange (I8I7-I878) sino con su hermano, el arquitecto Ludwig Lange, quien llegó a ser profesor de la

7. U Irich Thieme y Felix Becker, Allgemeines Lexikon der bildenden Künstler von der Antike bis zur Gegenwart, vol. xx, Leipzig, Verlag von E. A. Seemann, I927, p. 45. También en los periódicos Wiener Zeitung y Nerve Freie Presse del 3 de septiembre de 1895 y la revista Kunstchronik, NF, 1896, II, p. 43.

8. Kitlitschka, op. cit., p. 63. 
154

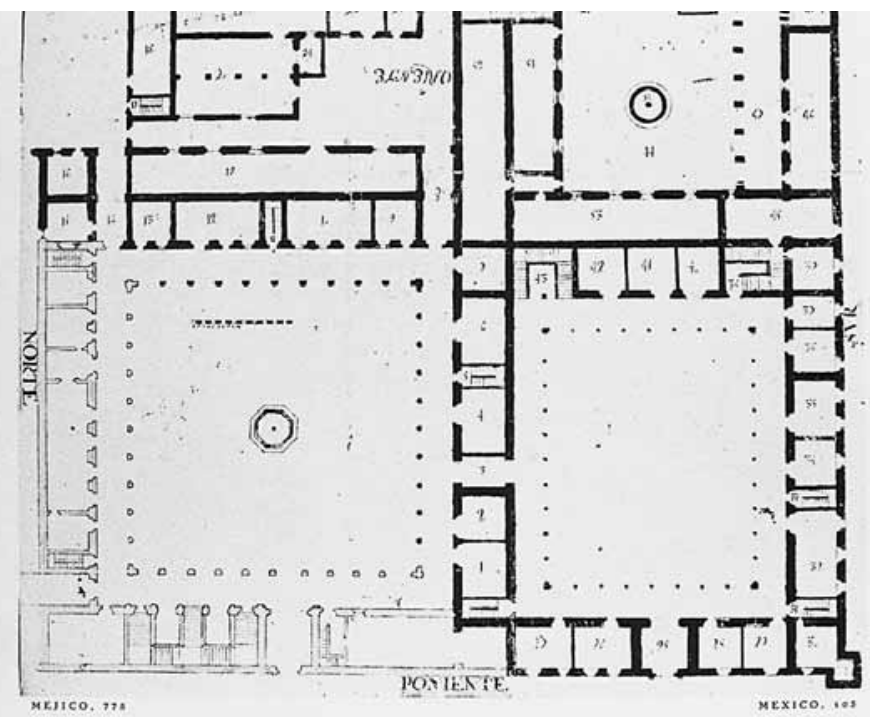

Figura 2. Palacio N acional, planta, 1705, Archivo de Indias de Sevilla.

Academia en 1847 , y cuyo primer periodo de creatividad se caracterizaba por edificios romano-bizantinos, seguido en el segundo por formas renacentistas, lo que repercutió en los planos mexicanos de K aiser y en sus obras austriacas, realizadas después de la caída del Segundo Imperio, y también analizadas por el propio doctor Vancsa. ${ }^{9}$

O bras de Carl Gangolf Kaiser y de otros alarifes, planos, dibujos y bosquejos

En M éxico se descubrieron unos planos, la mayoría de ellos firmados por "C. Kaiser" y otros autores, como E. Suban y Schafmann, y algunos aprobados por el entonces prefecto del alcázar de C hapultepec, Schaffer, en la colección O rozco y Berra del Instituto de G eografía y M eteorología. El estudio de estos documentos que se refieren al $\mathrm{C}$ astillo de Chapultepec y al Palacio $\mathrm{Na}$ cional fue la base para esta investigación.

Kaiser nos dejó anteproyectos ampliamente elaborados para la remodela-

9. Eckart Vancsa, "Zu C arl Gangolf Kayser. Ein W iener Architekt des Späthistorismus", en ARX-Burgen and Schlösser in Bayern, Ö sterreich und Südtirol, 3-4, 1980, pp. II-I7. 
Figura 3. Carl Gangolf Kaiser, capilla para el Palacio N acional, I866, legajo is65.

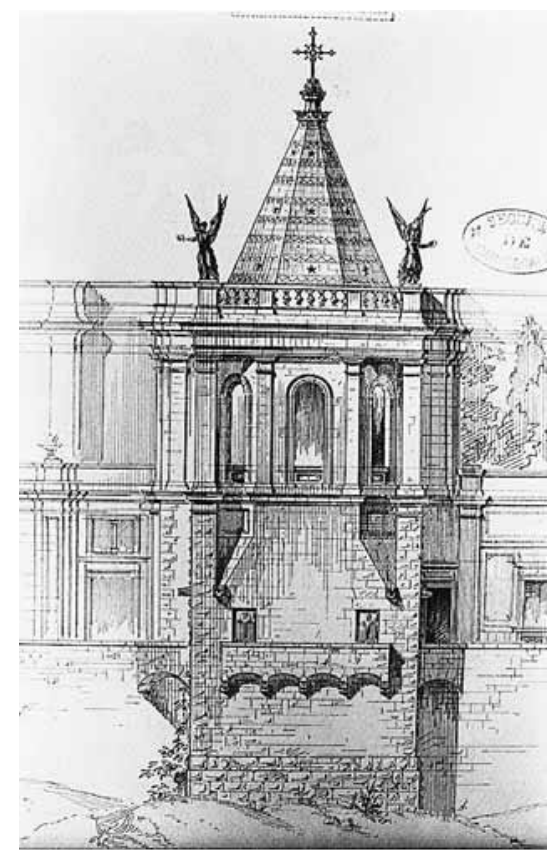

ción del Palacio $\mathrm{N}$ acional, claramente identificables por la firma y el estilo de dibujo, tan semejante al del arquitecto Julius H ofmann, quien diseñó los interiores del palacio de M iramar y quien debería adaptar el Palacio N acional de la ciudad de M éxico como residencia imperial. ${ }^{\text {Io }}$ Si bien las ideas arquitectónicas de Kaiser eran impresionantes y grandilocuentes, no pudieron realizarse debido a la falta de dinero; su concepto no habría encajado en el contexto urbano de la plaza principal de la ciudad de M éxico, cuyo elemento dominante, las torres de $\mathrm{C}$ atedral, hubiera recibido una competencia por parte de las numerosas torres del Palacio N acional remozado, con lo cual se hubiera perdido la jerarquía clara de los volúmenes. Sin lugar a dudas, este diseño poseyó una cualidad arquitectónica excelente; sin embargo, hubiera roto la escala del Zócalo y falsificado el edificio de la época colonial con un vestido europeizante del siglo XIX.II

Io. Eggert, op. cit., p. 75 .

II. M ichael D rewes, "Proyectos en el Imperio", en Palacio N acional, M éxico, 1976, pp. $20 \mathrm{I}-208$. 


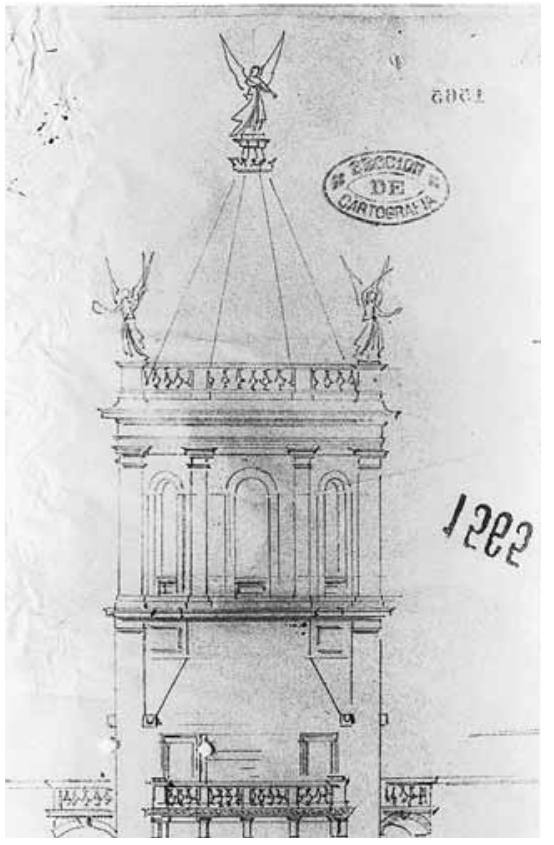

Figura 4. Carl Gangolf Kaiser, capilla para el Palacio $\mathrm{N}$ acional, bosquejo preliminar, legajo 1565 .

El arquitecto Lorenzo de la H idalga (1810-1872) proyectó una capilla para el Palacio $\mathrm{N}$ acional, y Kaiser hizo lo mismo, aunque no se sabe a ciencia cierta si su proyecto era también para este mismo edificio o para el Castillo de Chapultepec. Una perspectiva a lápiz, sin embargo, sugiere más bien esta última posibilidad, en vista de estar adosado al "caballero alto" del parterre de Chapultepec.

El núcleo original del Castillo de Chapultepec, un palacio neoclásico construido por el ingeniero Manuel Agustín Mascaró (I748-I8I2) de i785 a I787, en tiempos del virrey Bernardo de Gálvez, en el lugar de una ermita dedicada a san Francisco Javier, sufrió grandes estragos durante los bombardeos del I2 y i3 de septiembre de i847 por parte del invasor norteamericano, y estuvo sujeto a modificaciones y ampliaciones posteriores.

El archiduque Fernando Maximiliano de $\mathrm{H}$ absburgo eligió el Castillo de Chapultepec como residencia oficial, como antes de él lo habían acostumbrado los presidentes mexicanos. Santiago Rebull (ı829-1902), quien había sido nombrado pintor de cámara del emperador en I864, realizó en i866 cuatro Bacantes al estilo pompeyano en los pasillos a cubierto que rodean el par- 


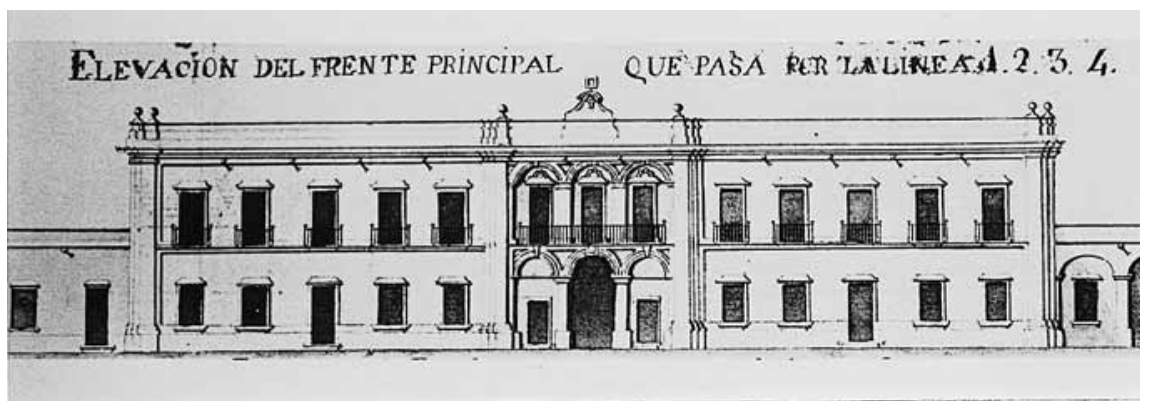

Figura 5. El palacio de Chapultepec, proyecto del Ing. M anuel Agustín M ascaró, I785-1787, Archivo de Indias de Sevilla.

terre, o sea, el jardín elevado de la época colonial del Palacio de Chapultepec $^{\mathrm{I2}}$ que fue remodelado y ampliado por el arquitecto mexicano Ramón Rodríguez Arrangoiti. El palacio colonial recibió unas arcadas y un vestíbulo en el frente y un ala lateral.

El arquitecto Ramón Rodríguez Arrangoiti (I830-1882) era alumno del $\mathrm{H}$. Colegio M ilitar en el Castillo de Chapultepec durante la intervención americana, y fue herido en su defensa el is de septiembre de i847. En i855 obtuvo el grado de doctor en matemáticas por la U niversidad de Roma, y de regreso a M éxico convirtió el ruinoso Castillo de Chapultepec en Alcázar Imperial en su calidad de arquitecto de la corte de M aximiliano, durante los años de I864 a I865. Construyó muchas obras en la ciudad de M éxico, como el H otel Gillow, en el centro, y el monumento a los $\mathrm{N}$ iños H éroes de i847; y, en otros lugares de la República, el templo de San José de Iturbide en el estado de Guanajuato y el Palacio de Gobierno de Toluca. En i87o presentó un proyecto para una nueva basílica en dicha ciudad. ${ }^{13}$ Rodríguez Arrangoiti diseñó también una capilla y unos templetes de acceso que, al igual que los proyectos de Kaiser, se quedaron en boceto.

Eleuterio M éndez (I830-1892) nació y murió en la ciudad de M éxico, donde realizó todos sus estudios y se graduó de ingeniero. Actuó como director de construcción del Ferrocarril del Valle y del Ilamado Circuito de Baños.

I2. N anda Leonardini, El pintor Santiago Rebull. Su vida y su obra (I829-I902), M éxico, U niversidad N acional Autónoma de M éxico, 1983, pp. 213-214.

I3. "Rodríguez Arrangoity, Ramón", en D iccionario Porrúa de historia, biografía y geografía de M éxico, t. III, P-Z, M éxico, Porrúa, I994, p. 2493. 


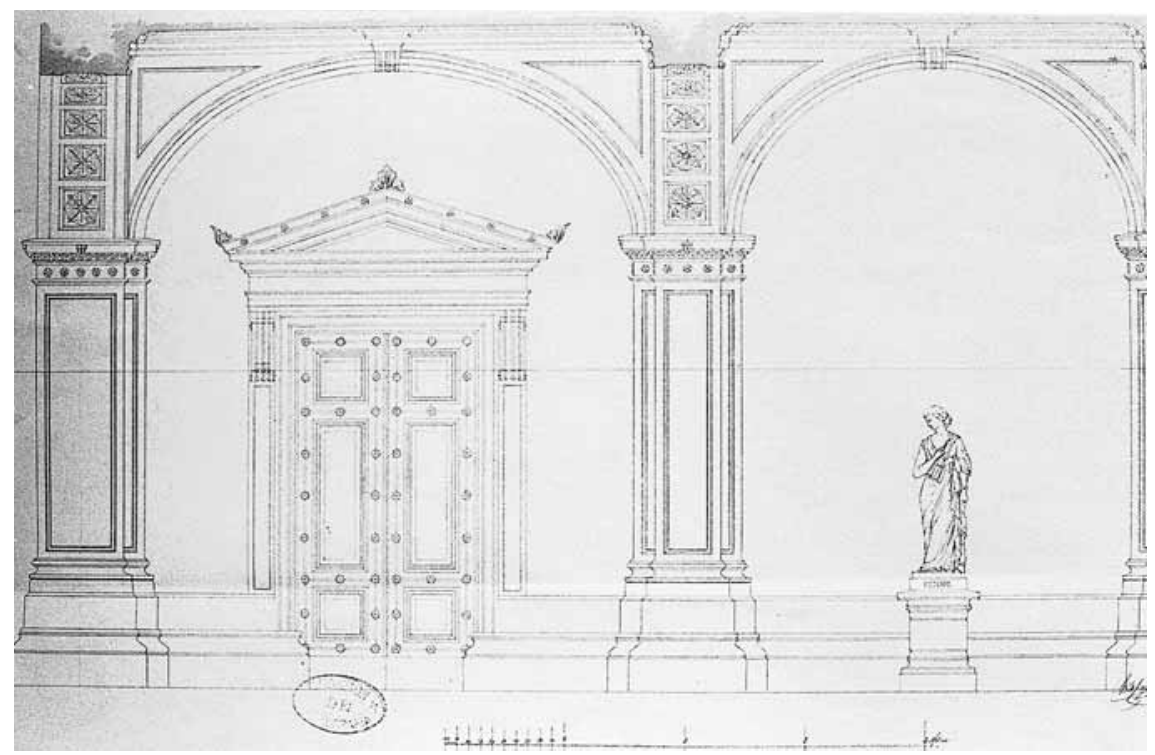

Figura 6. Interior del Palacio de Chapultepec, autor desconocido, salón con escultura de Euterpe, plano io3, legajo is64.

Fue profesor y subdirector de la Escuela Nacional de Ingeniería. ${ }^{\mathrm{I}}$ Estuvo frente a las obras del Castillo de Chapultepec como "ingeniero subdirector de las obras", y existen algunos dibujos suyos de elementos de instalaciones y de perfiles para escaleras para hacerse en hierro fundido.

El emperador M aximiliano había previsto un verdadero programa de renovación urbana de la ciudad de M éxico que no sólo se ocupaba del aspecto estético, como amplios parques, espaciosas plazas y anchas avenidas que parecen haber sido una tendencia dominante en la planeación del Imperio, sino también de problemas como canalización, abastecimiento de agua, alumbrado público, numeración de las casas, bomberos, higiene de los mercados y asuntos similares. H umilde es la cantidad de frutos de esta planeación, a veces presente en mínimos restos, lo que se explica por el corto periodo que duró el Imperio, la falta de recursos monetarios y la negligencia por parte de gobiernos republicanos posteriores. Entre dichos restos se cuentan, haciendo excepción de las insignias y de los símbolos imperiales: el Salón de los Emba- 


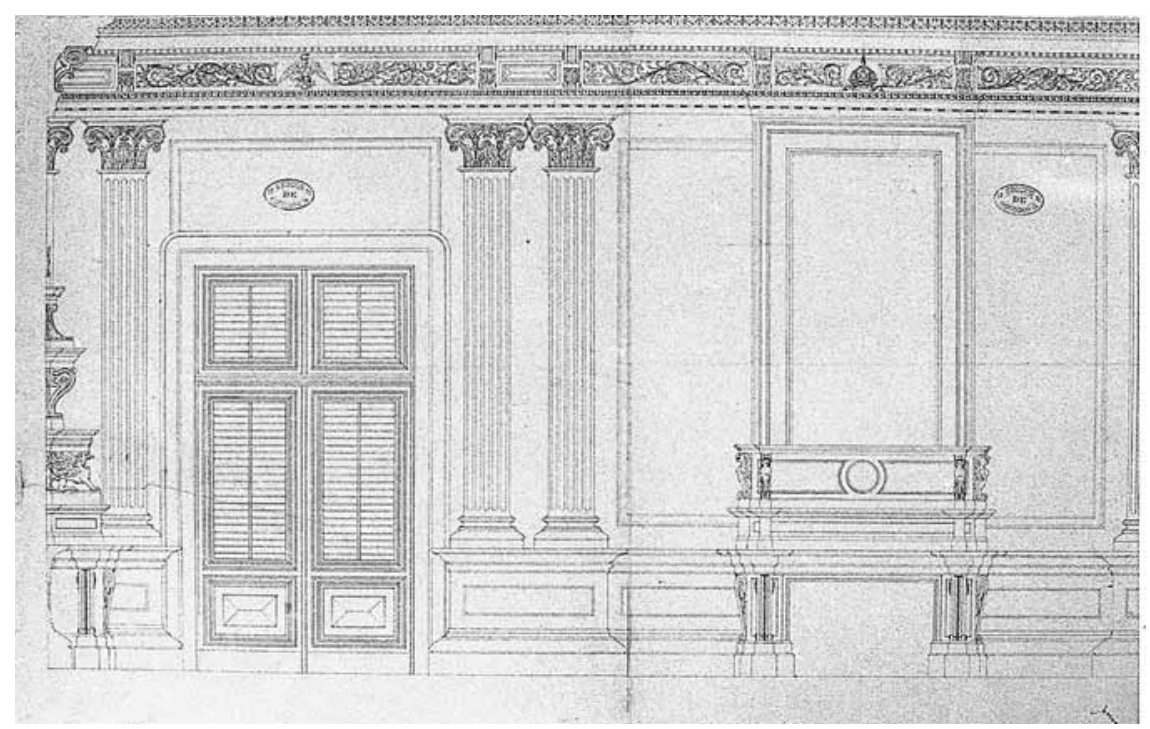

Figura 7. Interior del Palacio de Chapultepec, salón con espejo, autor desconocido, siglo xIx.

jadores del Palacio N acional; la avenida que conecta la capital con el C astillo de Chapultepec, antes Calzada de los Emperadores, hoy Paseo de la R eforma; partes de la apariencia externa de Chapultepec que remontan al arquitecto Ramón Rodríguez Arrangoiti, las pinturas pompeyanas de Santiago Rebull, los estanques de agua y el jardinado de la Plaza M ayor, así como la ampliación de la Alameda (la idea latinoamericana de convertir las plazas en arboledas parece originarse a causa de la iniciativa de $\mathrm{M}$ aximiliano) y la construcción de un nuevo Teatro $\mathrm{N}$ acional. ${ }^{\text {Is }}$

Kaiser, en particular, proyectó el remate del cuerpo central, con reloj y campanario, del palacio virreinal del Alcázar de Chapultepec, una gloriette parecida a la de los jardines del palacio de Schönbrunn de V iena, varias casas particulares cuya ubicación es imposible reconstruir, ya que nada de sus diseños llegó a realizarse. ${ }^{16}$ Además existen anteproyectos y bosquejos de Kaiser

I5. Johann Lubienski, D er maximilianeische Staat. M exiko I86I-1867, Viena, Köln, G raz, Böhlau Verlag, 1988, pp. 76-77.

I6. D rewes, "Proyectos de remodelación del Palacio de Chapultepec en la época del empe rador M aximiliano", en Anales del Instituto de Investigaciones Estéticas, núm. 5I, M éxico, 1983, pp. 73-82. 


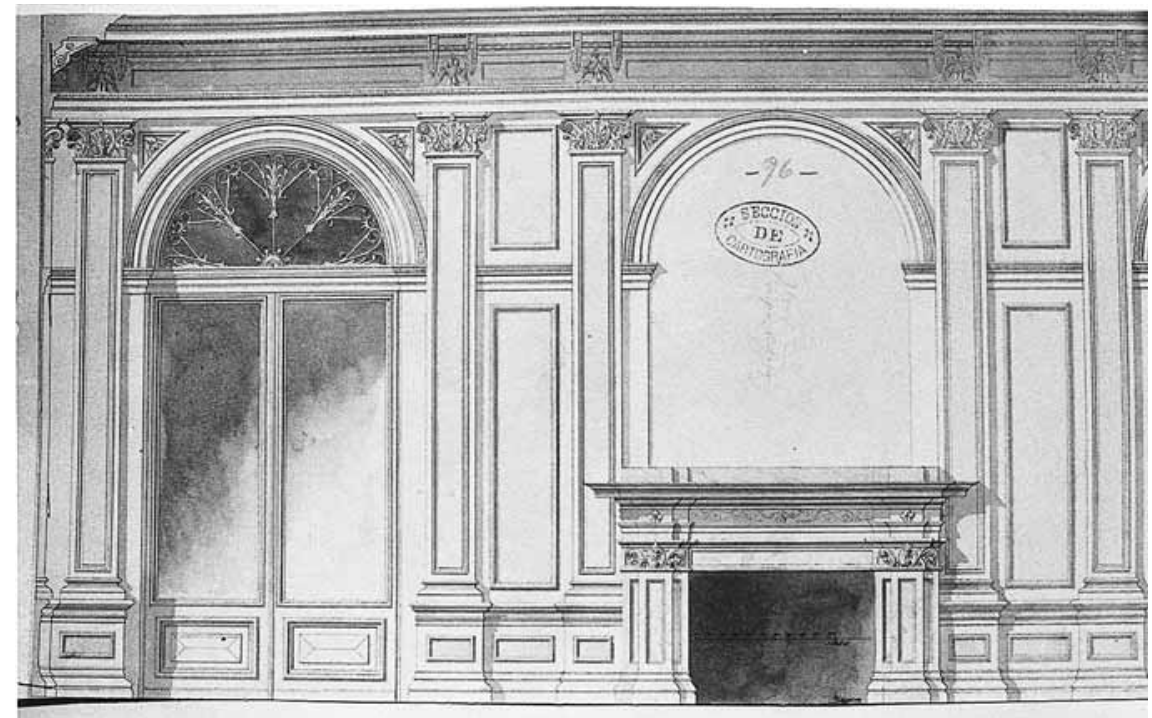

Figura 8. Interior del Palacio de Chapultepec, salón con chimenea, autor desconocido, plano 95, legajo 1564 .

en el mismo Instituto de G eografía y M eteorología u O bservatorio de Tacubaya, conocido popularmente como Casa Amarilla, que deberían de incluirse en un catálogo razonado de sus obras completas.

La investigación sobre los proyectos de remodelación del Palacio N acional y del Alcázar de C hapultepec salió también en un artículo publicado en alemán. ${ }^{17}$

\section{D ocumentos acerca de Kaiser y otros alarifes}

Además del descubrimiento de los planos originales de la época, el hecho de hallarse en el Archivo D oméstico de la Corte y del Estado, $\mathrm{H}$ aus, $\mathrm{H}$ of- und Staatsarchiv de Viena unos documentos relativos al arquitecto Kaiser en el Archivo de M aximiliano puede verse como una circunstancia muy feliz, ya

17. D rewes, "Projekte Carl Gangolf Kaysers für Kaiser M aximilian von M exico", en ARXBurgen and Schlösser in Bayern, Ö sterreich and Südtirol, 1980, 3-4, pp. 3-10. 


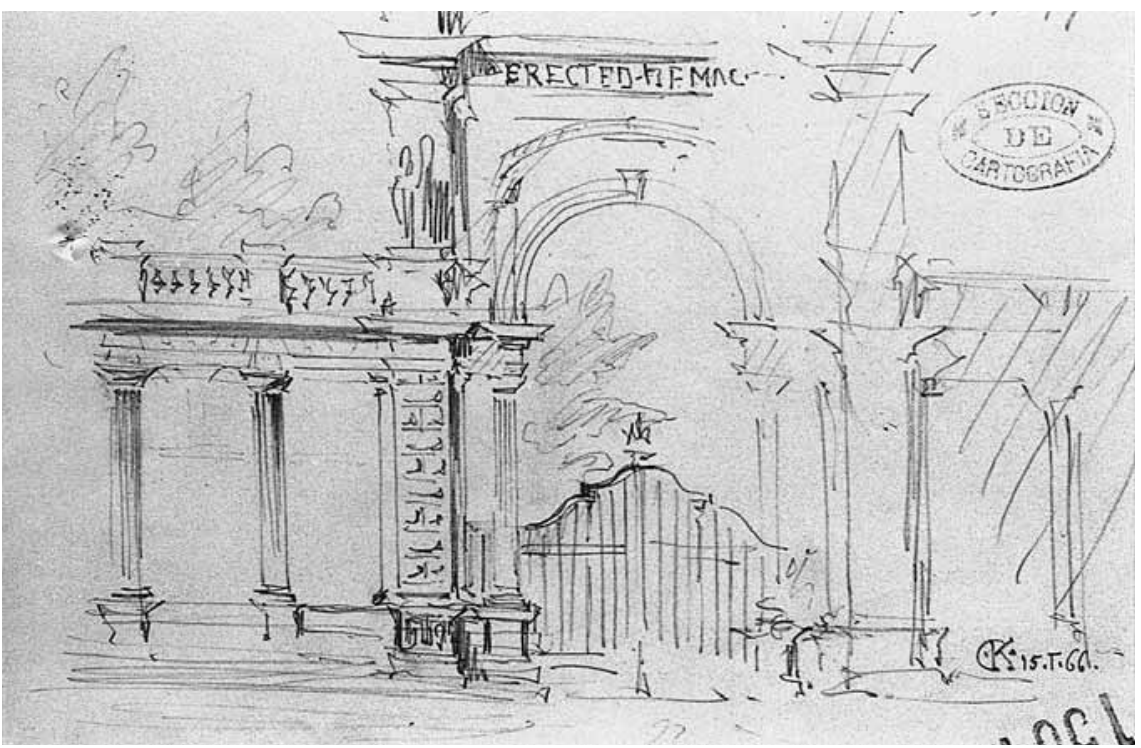

Figura 9. Carl Gangolf Kaiser, puerta principal de la Rotonda, I866, legajo 1564, plano 47.

que aportó más datos importantes. ${ }^{18}$ Se trata de una colección de cartas, facturas, boletos de diligencia, especificaciones y órdenes. Destacan una carta fechada el 24 de enero de i866, en la que se describe la remodelación de la Casa Borda'9 en Cuernavaca para la adaptación como residencia del archiduque $M$ aximiliano y de su cortejo, y en la cual se menciona también al arquitecto Kaiser, quien "no tuvo descanso durante toda la noche, y al amanecer había ya hecho cantidad de dibujos para la restauración futura y comenzó con el levantamiento de la casa [de Cortés]". Todavía no se han encontrado dichos dibujos, y sería interesante para un proyecto de investigación futuro el tema de la remodelación del palacio de C ortés en Cuernavaca por Kaiser.

0 tro documento importante es un oficio redactado por el propio Kaiser el is de mayo de i866 y dirigido al emperador Maximiliano, en el cual trata de convencer a éste de las ventajas de una organización lógica y coherente de

I8. Agradezco al doctor Johann Lubienski el haberme enviado fotocopias de dichos documentos para poder estudiarlos.

19. Eggert, op. cit., pp. 75-76. 


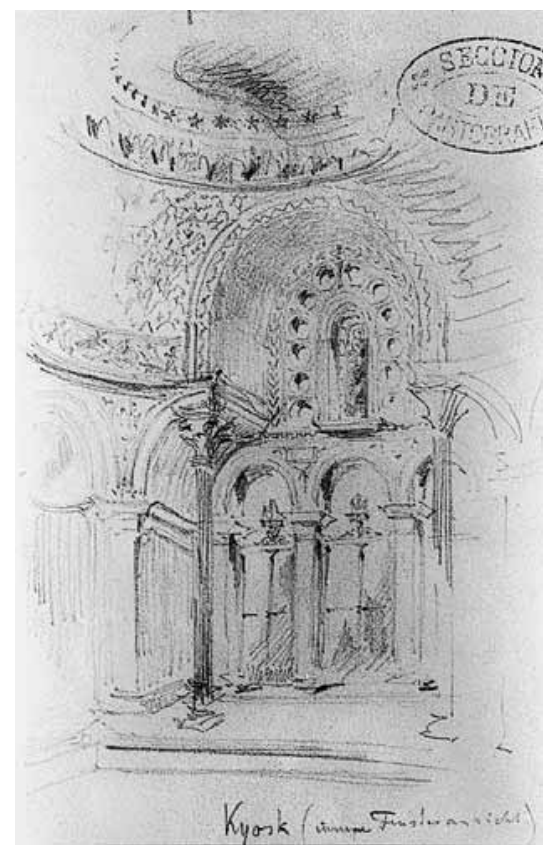

Figura ro. Carl Gangolf Kaiser, quiosco, 1866.

las obras públicas que, al parecer, se había dejado más o menos al azar. De por sí, Francisco de Paula de Arrangoiz, nacido en i8ı2, y por tanto contemporáneo de M aximiliano, criticó acremente la catastrófica y desatinada administración del Imperio. ${ }^{20}$

Los resultados de la investigación sobre estos documentos se publicaron en español ${ }^{21}$ y en alemán. ${ }^{22}$

20. Francisco de Paula de Arrangoiz, M éxico desde 1808 hasta I867, M éxico, Porrúa, I968, pp. 590, 736-74I.

2I. D rewes, "C arl Gangolf Kaiser (I837-I895), arquitecto de la corte del emperador M aximiliano", en Anales del Instituto de Investigaciones Estéticas, núm. 59, M éxico, 1988, pp. 239-254.

22. D rewes, "C arl Gangolf Kaiser (1837-1895) und seine Tätigkeit als H ofarchitekt M aximilians von M exiko", en M itteilungen des Instituts für Ö sterreichische G eschichtsforschung, 1993, 24, pp. 383-403. Aprovecho la ocasión para agradecer al doctor Konrad Ratz el haber hecho posible la publicación de este artículo. 


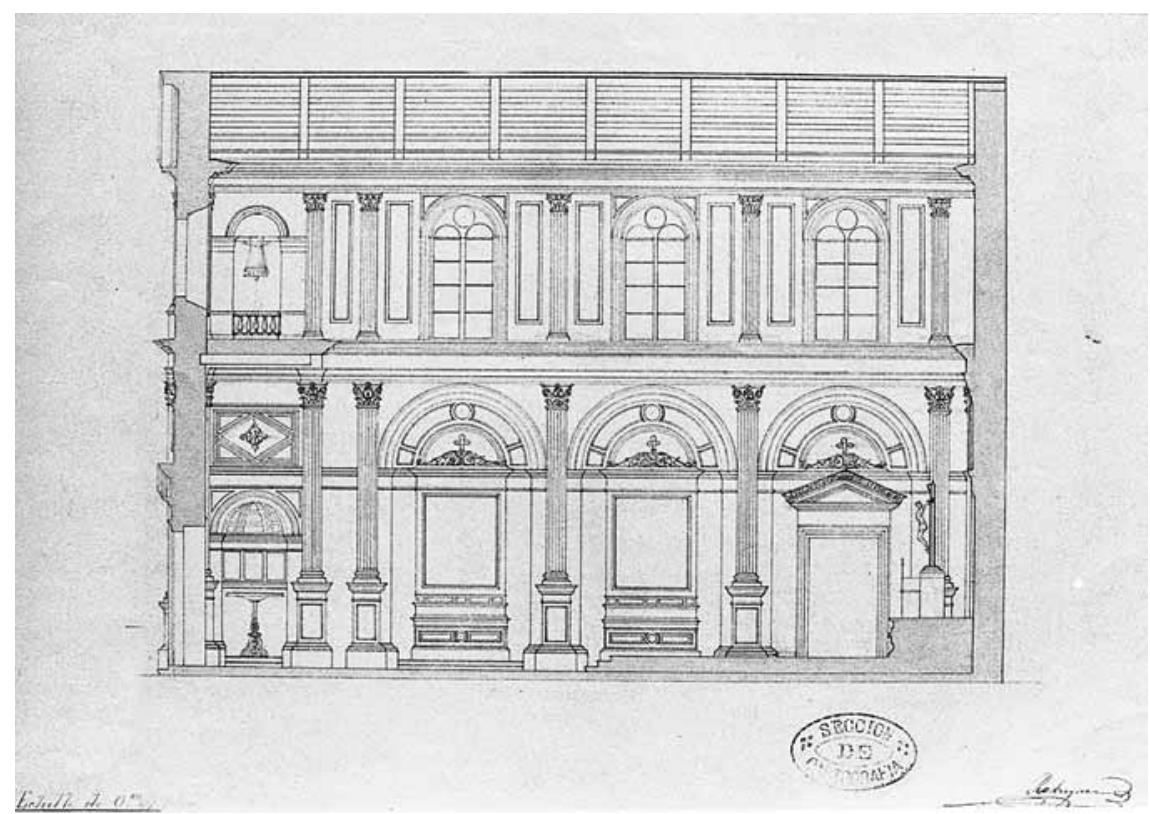

Figura II. Ramón Rodríguez Arrangoiti, capilla para Chapultepec, cortelongitudinal, legajo 1564.

\section{El busto-retrato de Carl Gangolf Kaiser y valoración}

También se dio la circunstancia feliz de que existe un busto-retrato del arquitecto Kaiser ${ }^{23}$ en mármol originario de Salzburgo, hecho por el escultor Victor O skar Tilgner (1844-1896) y que se conserva en el castillo de Kreuzenstein que el propio Kaiser restauró. Representa al alarife, un hombre maduro, en atuendo medieval de acuerdo con el gusto historicista de la épo$\mathrm{Ca}^{24}$ y la inscripción reza en caracteres góticos en la base: "K arl Gangolf Kayser". $^{25}$

Con la reserva de que habría que delimitar el concepto de "Io mexicano",

23. Varios autores, M aximilian von M exico I832-1867, Viena, Verlag Enzenhofer, I974, p. 250. Véase también $V$ ancsa, op. cit., p. I3.

24. D rewes, "El busto del arquitecto Carl Gangolf Kaiser", en Anales del Instituto de Inves tigaciones Estéticas, núm. 63, M éxico, 1992, pp. I77-I8I.

25. D rewes, "W eitere N otizen über den Architekten Carl Gangolf Kaiser", en Festschrift $\mathrm{M}$ artin Grassnick, 1997, pp. 4I-43. 


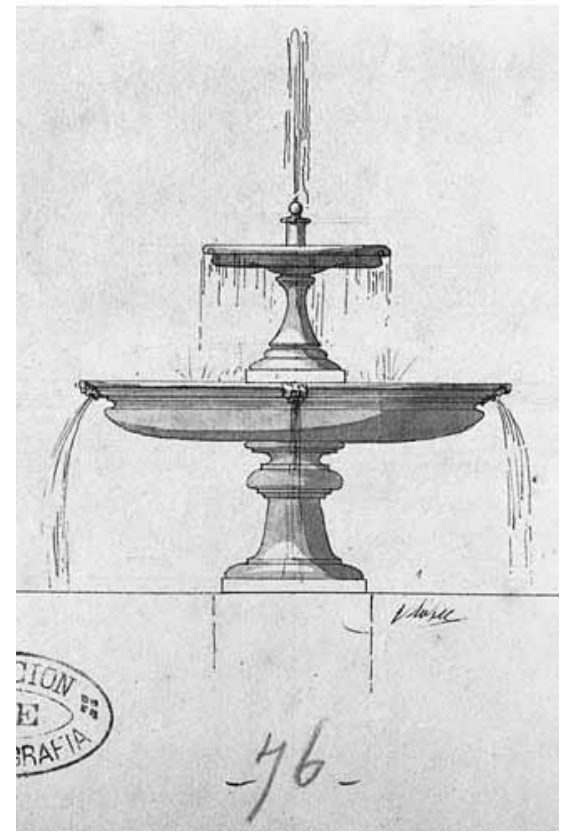

Figura I2. Autor desconocido, fuente, plano 76, legajo 1564.

podemos afirmar que las obras del arquitecto Kaiser y de sus colegas austriacos y mexicanos se hallan lejos de expresar un carácter propio, y mucho menos propiciaron una definición del Estado nacional mexicano. Ya antes del Segundo Imperio se dio en M éxico el fenómeno de afrancesamiento en la cultura del país, que se manifestó en la imitación de patrones europeos, especialmente franceses, en los modales y en las artes plásticas.

Como escribí de una manera generalizante: ${ }^{26}$

A la mayoría de estos proyectos comentados, les es propio cierto aire de sueño; son engendros fantásticos que manifiestan en el campo de las artes plásticas la faIlida aventura de establecer desde la lejana Europa un imperio en M éxico, una hazaña que con justa razón puede relegarse al país de los sueños, en vista de lo anacrónico del intento, ya que se trataba de implantar formas de vida ajenas a la realidad nacional, y un modo de gobernar que ya no era el adecuado en una época, en la cual iba consolidándose la democracia liberal y republicana. 
Figura i3. Autor desconocido, jaula para ratoncitos.
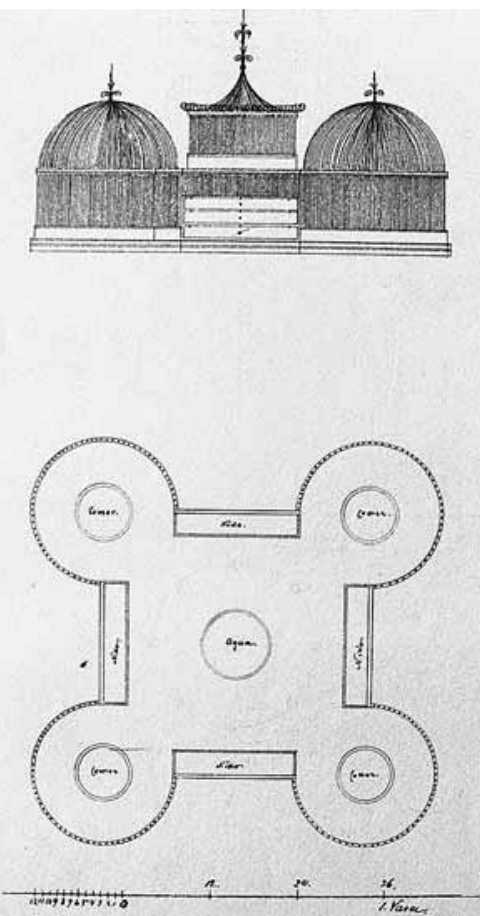

0 mi caracterización de Kaiser:27

Si bien era un arquitecto de muy buen gusto en el diseño y muy capaz de plasmar de manera convincente en el papel sus ideas, sus propuestas de remodelación, tanto del Palacio N acional como del alcázar de Chapultepec, hubieran desvirtuado considerablemente el carácter de estos edificios. Pero él era obviamente una presa del historicismo ecléctico tardío europeo, y después de la caída del Imperio logró alcanzar en sus obras austriacas mayor sensatez y adaptación a la dignidad de los edificios que restauró, tal vez debido también a su proceso natural de maduración. En M éxico estaba a punto de forzar los neoestilos - no se sabe hasta dónde K aiser sufriera las influencias de Viollet le D uc (I8I4-I879) - sobre construcciones netamente coloniales, si sus proyectos se hubieran realizado,

27. D rewes, "C arl Gangolf Kaiser... ", art. cit., p. 254. 
DOI: http://dx.doi.org/10.22201/iie.18703062e.2000.77.1949

166

MICHAEL DREWES

mientras que en Austria su actitud era más acorde con el carácter de la arquitectura de ese país.

M ucho después de la restauración de la República, la fase que siguió al fallido Imperio de $M$ aximiliano, surgieron manifestaciones nacionalistas en el arte. La época colonial era un tema difícil de tratar, por razones evidentemente políticas, por lo que se recurrió a temas de la historia prehispánica.

El escultor M anuel Vilar (I812-1860) inició el interés escultórico por los héroes indígenas, que culminó con el Monumento a Cuauhtémoc (1887) de Francisco M . Jiménez y M iguel N oreña. Uno de sus discípulos fue Felipe Sojo, quien modeló un busto de M aximiliano en I864. Rodrigo Gutiérrez pintó El senado de T laxcala, y José O bregón, El descubrimiento del pulque, que son obras donde se intenta captar el tema prehispánico, pero tratando de establecer una equivalencia con la Antigüedad clásica, por lo cual es difícil clasificarlas como manifestaciones netamente nacionalistas. ${ }^{28}$

\section{Recomendaciones para investigaciones futuras}

Lo que falta hacer es profundizar más la biografía de Kaiser y analizar más a fondo sus obras austriacas. Todavía quedan por reseñarse muchos bosquejos y anteproyectos del propio Kaiser que se encuentran en el archivo del Instituto de Geografía y M eteorología, Sección de Cartografía, del O bservatorio Astronómico $\mathrm{N}$ acional de Tacubaya, que consisten en planos preliminares de casas particulares, rampas y plazas de acceso para el Alcázar de C hapultepec y diseños de fuentes, monumentos y accesorios del parque que lo rodea. \&

28. Justino Fernández, "EI siglo romántico. El arte de M éxico en el siglo XIX”, en Cuarenta siglos de plástica mexicana. Arte moderno y contemporáneo, M éxico, Herrero, I97I, pp. 38, $59-60$. 
DOI: http://dx.doi.org/10.22201/iie.18703062e.2000.77.1949

OTRAS APROXIMACIONES A CARL GANGOLF KAISER

\section{Fuentes}

Anders, Ferdinand, D ie Gärten M aximilians, Viena, 1987.

Arrangoiz, Francisco de Paula de, M éxico desde I808 hasta I867, M éxico, Porrúa, 1968.

D iccionario Porrúa de historia, biografía y geografía de M éxico, M éxico, Porrúa, I994.

D rewes, M ichael, “Proyectos en el Imperio”, en Palacio N acional, M éxico, 1976, pp. 20I-208. , "Projekte Carl G angolf Kaysers für Kaiser M aximilian von M exico", en ARX-Burgen and Schlösser in Bayern, Ö sterrèch and Südtirol, I980, 3-4, pp. 3-ı. , "Proyectos de remodelación del Palacio de Chapultepec en la época del emperador M aximiliano", en Anales del Instituto de Investigaciones Estéticas, núm. 5I, M éxico, I983, pp. 73-82.

, "Carl Gangolf Kaiser (I837-1895), arquitecto de la corte del emperador M aximiliano", en Anales del Instituto de Investigaciones Estéticas, núm. 59, M éxico, 1988, pp. 239-254. , "El busto del arquitecto Carl Gangolf Kaiser", en Anales del Instituto de InvestigacionesEstéticas, núm. 63, M éxico, 1992, pp. I77-18I.

—, "W eitere N otizen über den Architekten Carl G angolf Kaiser", en Festschrift M artin Grassnick, 1997, pp. 41-43.

Eggert, Klaus, "M aximilian und seine Kunstschöpfungen", en M aximilian von M exiko I832I867, Viena, Enzenhofer, 1974, pp. 66-78.

Fernández, Justino, "El siglo romántico. El arte de M éxico en el siglo XIX", en Cuarenta siglos de plástica mexicana. Arte moderno y contemporáneo, M éxico, H errero, I97I.

Kitlitschka, Werner, "Erzherzog Ferdinand M aximilian und die bildende K unst", en M aximilian von M exiko I832-I867, Viena, Enzenhofer, I974, pp. 58-65.

Leonardini, N anda, El pintor Santiago Rebull. Su vida y su obra (I829-I902), M éxico, U niversidad N acional Autónoma de M éxico, 1983.

Lubienski, Johann, D er maximilianeische Staat. M exiko I86I-1867, Viena, Köln, Graz, Böhlau Verlag, 1988.

M üllner, Franz, "Johann C arl Fürst Khevenhüller-M etsch, ein Kampfgefährte Kaiser M aximilians von M exiko", en M aximilian von M exiko I832-I867, Viena, Enzenhofer, 1974, pp. I36-i62.

Springer, Elisabeth, "M aximilians Persönlichkeit", en M aximilian von M exiko I832-I867, Viena, Enzenhofer, 1974, pp. 12-23.

Thieme, Ulrich y Felix Becker, Allgemeines Lexikon der bildenden Künstler von der Antike bis zur G egenwart, vol. xx, Leipzig, Verlag von E. A. Seemann, 1927.

Vancsa, Eckart, "Zu Carl Gangolf Kayser. Ein W iener Architekt des Späthistorismus", en ARX-Burgen und Schlösser in Bayern, Ö sterreich and Südtirol, I980, 3-4, pp. II-I7. 